\title{
Erkenntnis zwischen Erfühlen, Intuition und Anschauung
}

Renate Bräuninger

\section{Die Verbindung zwischen Innen und Außen}

$\mathrm{Zu}$ den Sens(a)tions, die durch und im Tanz erfahrbar werden, gehören die Unmittelbarkeit der körperlichen Erfahrung beim Tanzen, intuitive künstlerische Entscheidungsfindungsprozesse beim Improvisieren und Choreografieren sowie die affektive und emotionale Resonanz, welche die $\mathrm{Zu}$ schauenden bei einer Aufführung spüren. Bei diesen Erfahrungen besteht eine Verbindung zwischen äußerer Wahrnehmung und innerer Empfindung. Zum Bereich des inneren Empfindens gehören für die Tanzenden sowohl das Erinnern von Schrittfolgen durch das sog. Muskelgedächtnis (was eigentlich eine Verbindung zwischen Nervenenden und Gehirnregionen ist), als auch eine gewisse kinästhetische Empathie für jene, die sich mit ihnen auf der Bühne oder im Studio befinden. Für das Publikum gehört dazu kinästhetische und affektive Resonanz, welche eine Aufführung in ihnen auslöst. Zum Bereich des äußeren Wahrnehmens gehört für Tanzende sowie Publikum all das, was bei einer Aufführung oder bei einer Probe beobachtet werden kann, wie z.B. Form und Gestalt einer Bewegung. Tänzer*innen und Choreograf*innen nutzen im Probenprozess Verbindungen zwischen beiden Bereichen, wenn sie nach motorischen Quellen von Bewegungen suchen, damit Bewegungen nicht nur eine bestimmte Wirkung, sondern auch eine überzeugende Gestalt haben. Bei einer Aufführung entstehen solche Verbindungen, wenn Zuschauende versuchen, das Gesehene sowohl durch die beredte Gestik der Bewegung als auch durch Affekte und Emotionen, die bei ihnen ausgelöst werden, zu interpretieren. 
Diese Formen der Wahrnehmung und Erkenntnisgewinnung sind somit nie vollkommen frei von Subjektivität, weshalb sich auch die geisteswissenschaftliche und künstlerisch-wissenschaftliche von der naturwissenschaftlichen Forschung unterscheidet. Letztere ist durch eine Trennung zwischen dem Subjekt und seinen Empfindungswelten, seinem Inneren und der Welt, die im Verhältnis zum Individuum das Außen, das Objekt darstellt, charakterisiert. Damit verbunden ist die im Zuge der Aufklärung entstandene Annahme, dass objektive Wissensfindung nur durch die Unterscheidung vom Selbst als Subjekt, welches die Erkenntnis gewinnt und dem Objekt als Gegenstand, über das Erkenntnisse gewonnen werden, stattfinden kann. Mit der Aufklärung wird die Unmittelbarkeit der oben genannten Sens(a) tions durch Versuche, ihr Zustandekommen zu erklären, relativiert. Zwar finden sich persönliche Empfindungen im aufklärerischen Denken. Stimmung ist für Immanuel Kant eine wichtige Komponente beim Entstehen eines Geschmacksurteils. In seiner Kritik der Urteilskraft (1790) ist Stimmung oder Gestimmtheit der oder des Betrachtenden bei der Festlegung von Kriterien, die es erlauben, das Schöne und Erhabene zu beurteilen, von Bedeutung. Das Sinnliche und der Verstand müssen für ihn zusammenkommen:

Die Verbindung und Harmonie beider Erkenntnisvermögen, der Sinnlichkeit und des Verstandes, die einander zwar nicht entbehren können, aber doch auch ohne Zwang und wechselseitigen Abbruch sich nicht wohl vereinigen lassen, muß unabsichtlich zu sein und sich von selbst so zu fügen scheinen; sonst ist es nicht schöne Kunst. (Kant 2001: 212)

Aber Kant führt eine Schicht der Mittelbarkeit ein, in dem er die sensuelle Erfahrung als durch a priori gegebene Faktoren, ob als Kognition verstanden oder nicht, geformt sieht. Unsere Empfindung muss somit nicht der Wirklichkeit an und für sich entsprechen, sondern zeigt lediglich, wie der Mensch im Unterschied zum Tier die Welt wahrnimmt. Mit der Abhängigkeit der Wahrnehmung von einem a priori ist deren Unmittelbarkeit relativiert. Den romantischen Dichter Heinrich von Kleist stürzte diese Annahme in eine tiefe Krise. Er fragte sich, kann ich durch meine persönlichen Empfindungen zur Wahrheitsfindung gelangen, wenn diese durch meine körpereigene und mir zum Teil unerklärliche Wahrnehmungsmaschinerie bestimmt wird? Eine Kluft tut sich auf zwischen persönlicher Beobachtung und naturwissenschaftlichem Experiment, wobei letzteres als das zuverlässigere gilt. 
Doch wurde Objektivität in den Naturwissenschaften wirklich erreicht? Der Wissenschaftskritiker Paul Feyerabend weist in seinem Buch Against Method (1975) darauf hin, dass scheinbar objektive Experimente oftmals von älteren und subjektiveren Denkmodellen beeinflusst sind:

It is this historico-physiological character of the evidence, the fact that it does not merely describe some objective state of affairs but also expresses subjective, mythical and long-forgotten views concerning this state of affairs, that forces us to take a fresh look at methodology. (Feyerabend 2010: 47)

Dennoch errang im 19. Jahrhundert naturwissenschaftliches Denken an den Universitäten eine Vormachtstellung und zwang die geisteswissenschaftlichen Fächer zu einer Anpassung ihrer Methodologien. Am Beispiel der Musikwissenschaft wird deutlich, wie dies zu einer Abwendung vom sensuellen hin zu formalen Komponenten der Musik führt. Der Versuch einer Objektivierbarkeit von Musik sowie ihrer Vermittlung spiegelt sich auch in der Fokussierung auf Editionen, wie zum Beispiel Urtextausgaben und der Akustikforschung wider. Urtextausgaben, häufig auch Monumente der Tonkunst genannt, bevorzugen den Objektcharakter der musikalischen Partitur im Vergleich zu der Vergänglichkeit der Aufführung als Grundlage für Analysen. Von musikalisch gebildeten Hörer*innen wurde erwartet, sich eine Komposition als formales Tongebilde vorstellen zu können. Die Akustikforschung reduzierte musikalisches Hören auf ein physikalisches Phänomen. Diese Ansätze führten zwar zu der Entwicklung von Analysemethoden, die eine Erschließung von Einstudierungen und Aufführungen für Musiker*innen und Musikwissenschaftler*innen erleichtern, zum andern aber auch zu einer Vernachlässigung der Untersuchung von Aufführungstradition und -praxis und den hierbei involvierten oralen Praktiken. Ebenfalls wurde die sinnliche Erfahrung des Musikhörens wenig berücksichtigt (Karnes 2016). Erstmalig beschäftigte sich das CHARM Projekt (2009) in Großbritannien mit der Untersuchung und Analyse von Tonaufnahmen als Dokumente. Hierbei zeigte sich, dass Aufnahmen stärker von oral überlieferten Aufführungstraditionen als von einer Treue gegenüber dem Notentext beeinflusst waren. 


\section{Das Problem der Übersetzung in Sprache}

Tanzwissenschaftler*innen sollten deshalb bei dem Versuch, sich im Machtgeflecht der Bildungsideale als Disziplin mit besonderen Formen der Wissensfindung zu etablieren, nicht über ähnliche Fallstricke stolpern, wie es den Musikwissenschaftler*innen im 19. Jahrhundert passiert ist. Zentral scheint mir hier das Problem der Übersetzung in Sprache. Auf der Konferenz zum Thema Sens(a)tions wurde mehrfach angesprochen, wie schwierig es sei, die über Tanz gewonnenen Erkenntnisse in Sprache einzufangen und zu reflektieren. Einer der Gründe dafür ist, dass Tanz und Sprache unterschiedlich funktionieren. Die beschriebene Unmittelbarkeit der Erfahrung von Tanz und Bewegung unterscheidet sich von der Dar- oder Vorstellbarkeit, die Sprache zu ihrer Vermittlung braucht. Zur Kommunikation durch Sprache gehört die Verbindung von Wörtern und Begriffen mit einer bestimmten Vorstellung von Gegenständen und/oder mehr oder minder abstrakten Ideen. Selbst wenn das Objekt nicht aktuell vorhanden ist, kann es vorgestellt und somit analysiert und entziffert werden. Hieraus ergibt sich die Frage, inwieweit Vorstellungsvermögen in jedem Fall notwendig ist, um Erkenntnis zu gewinnen. Mit anderen Worten, inwieweit unsere Interpretation von Wahrnehmung darauf beruht, dass eine Darstellung eines Phänomens mental nachvollzogen und somit semiotisch entschlüsselt werden kann, was beim jetzigen Stand der Forschung nicht eindeutig geklärt ist. Trotzdem sollte Sprache als metadiskursives Modell hinterfragt werden »cognitive science [should] move beyond the perspective that the exclusive province of thought is language and towards the notion that language is but one of a range of human communicative resources« (Zbikowski 2012: 157). Denn im Unterschied zu Sprache braucht nicht alle Erkenntnis im Bereich Tanz Vorstellung, sondern sie kann auch direkt auf vorangegangene körperliche Erfahrungen zurückgreifen. ${ }^{1}$ Der sogenannte Textual-Turn und zu einem gewissen Grad auch noch der Performative-Turn in den Geisteswissenschaften, der den darstellenden Künsten einen Textcharakter zuschreibt, eröffnet scheinbar die Möglichkeit, objektiv Sinn und Bedeutung eines Kunstwerks erschließen zu können. Doch solche Methodologien werden unmittelbarer Erfahrung und Intuition nicht gerecht, weil durch sie, mit der Unterscheidung zwischen Be-

1 In diesem Zusammenhang sind auch die Kapitel 3 und 4 in Varela/Thompson/Rosch (1993) zu beachten. 
zeichnetem und Bezeichnenden, eine Mittelbarkeit eingeführt wird, die der zwischen Subjektivem und Objektivem gleicht, wie sie für das naturwissenschaftliche Denken charakteristisch ist. Die Unmittelbarkeit der sinnlichen Anschauung kann aber nicht allein durch diesen Blick von außen verstanden werden, sondern sie braucht auch ein Verständnis, der hier zugrunde liegenden körperlichen, sinnlichen Erfahrungen. Spätestens mit der Wende zum Prozess-Turn sollte der entziffernde Blick von außen aufgegeben werden, sonst unterscheidet sich die Darstellung von Prozessen nicht von der Kritik einer Aufführung und Prozesse sind nichts anderes als eine neue Form des Produkts.

Was das theoretische Verständnis der Unmittelbarkeit körperlicher Empfindungen angeht, so bietet die Phänomenologie als philosophische Disziplin hier Lösungsansätze, denn sie hebt die Gespaltenheit zwischen Subjekt und Objekt auf und schlägt eine Form der Wahrnehmung vor, in der das Subjekt mit der Welt verschmilzt. Es ist auffällig, dass Maurice Merleau-Ponty, sicher auch weil er Mediziner und Psychologe war, das Funktionieren unseres kognitiven Apparats durch unfall- und krankheitsbedingte Ausfallerscheinungen zu erklären versucht, womit er in gewisser Weise den Kreis zu Kant schließt, denn der kognitive Apparat als solcher bleibt weiter die objektive Grundlage des Erklärungsmodells. Merleau-Ponty geht davon aus, dass der Körper direkt und oft ohne Reflexion mit der Welt in Kontakt tritt. Problematisch bleibt im Kontext der Theaterwissenschaften, dass Merleau-Ponty eigentlich so gut wie nie auf einen Aufführungskontext hinweist, er erwähnt auch ganz selten einen Probenkontext, wie beim Beispiel des Organisten, hier sieht er den Körper als die Verbindung zur Welt:

Deutlicher noch zeigt das Beispiel der Instrumentalmusik, daß weder Cewohnheit im Denken, noch im objektiven Leib, sondern im Leib als weltvermittelndem sich gründet. Ein geübter Organist kann bekanntlich auch auf einer ihm unbekannten Orgel mit mehr oder weniger Klaviaturen und einer anderen Anordnung der Register als bei seinem gewohnten Instrument spielen. Eine Stunde Übung reicht zur Vorbereitung eines Konzerts hin. [...] Zwischen dem musikalischen Wesen des Stückes, wie es die Partitur vorzeichnet, und der wirklich um die Orgel herum klingenden Musik stiftet sich ein unmittelbarer Bezug, daß der Leib des Organisten und das Instrument nur mehr Durchgangsorte dieses Bezugs sind. (Merleau-Ponty 2005: 175) 
Merleau-Ponty weist hier auf die unmittelbare Begegnung des Körpers mit der Welt hin, wie sie Tanzschaffenden bekannt ist, der Körper spürt sich selbst und interagiert mit dem Raum, ohne darüber kontinuierlich nachzudenken. Der professionelle Kunstschaffende hat Routinen entwickelt für Proben und Auftritte, die automatisch zum Einsatz kommen, ohne dass diese jeweils durchdacht werden müssen. Für tanzwissenschaftliche Arbeitsweisen ist es wichtig, dass solche Erfahrungen, weil nicht objektivierbar, sich einer semiotischen Entschlüsselung entziehen und somit nicht mit einem der etablierten, im weitesten Sinn aufText basierenden, Modellen verstanden werden können.

\section{Intuitive Entscheidungsfindung im künstlerischen Prozess}

Künstlerische Prozesse beruhen auf komplexen Entscheidungen. Hier öffnet sich ein Feld, das zwischen intuitivem Wissen, Reflexion und Theoriebildung angesiedelt ist, wo ein Gefühl für das Passende, mit einem Nachdenken darüber, warum dies der Fall ist, und mit einer Einordung in vorhandenes Wissen verknüpft wird. Wenn ein/e Choreograf*in überlegt, an welche Stelle in einer Choreografie eine Bewegungssequenz am besten passt, spielt ein bestimmtes Urteilsvermögen eine Rolle, das aus einer Mischung aus Empfinden und Reflexion besteht, einer Form der ästhetischen Intuition. Eine Bewegung sollte sich körperlich richtig anfühlen und gut in der Aufführung ausgeführt werden können. Vergleicht die oder der Tanzschaffende die Sequenz mit ähnlichen Bewegungsfolgen in anderen Choreografien, so wirkt sie gelungen und funktioniert im Kontext. Es stellt sich ein Empirisches »das passt« ein. Das Passende könnte mit dem, was Kant als das Kunstschöne beschreibt, vergleichbar sein, das, was für Kant auf der unmittelbaren Anschauung beruht und keinen Begriff braucht (Kant 2001: 25). Langjährige Erfahrung, ein bestimmtes handwerkliches Können und die Fähigkeit, darauf intuitiv zu reagieren und darüber zu reflektieren, spielen hier zusammen. Diese Prozesse beinhalten eine Kenntnis der Praxis und ein Verständnis von Metapraxis:

It has nothing at all to do with impulse, with the sinstinctives, nor is it located on the other side of the rational; nor indeed, as I have indicated elsewhere, is the expertintuitive just intuitive . On the contrary, expertintuitive mas- 
tery has internalized performer-specific and performance-specific analysis and deliberation, as this has been experienced by the individual practitioner, generally in a collaborative set-up; it involves a dynamic and often unstable feedback loop. It is key to decision making in professional practice and it has internalized aspects of performance metapractice developed in the context of different stagings. (Melrose 2018: 156)

Die Frage ist, wie dergleichen Erfahrungen verbalisiert werden können und ob der traditionelle tanzwissenschaftliche Diskurs dazu ausreicht, z.B. in der Tanzkritik, der Bewegungsanalyse und der Darstellung tänzerischer Praxis. Vor allem die Practice as Research-Forschung im angelsächsischen Raum hat gezeigt, dass eine Reihe von diskursiven Strategien und Arten des Schreibens, wie zum Beispiel Tagebucheinträge, Prosa-Darstellungen von künstlerischen Probensituationen neben Reflexionen über Prozesse stehen können. Vielleicht sollte anstelle etablierter Konventionen des Schreibens, eine Fülle von Darstellungsweisen gesetzt werden! Es sollte aber in jedem Fall ein Bewusstsein dafür herrschen, welche Strategie für welchen Zweck die geeignetste ist. Mit den neuen Medien entsteht auch die Frage, inwieweit nicht eine multimediale Form sensuell subjektive Erkenntnisse besser einfangen kann. Hier sei zum Beispiel die Motion Capture Technologie angeführt, die Reaktionen der Tänzer aufeinander darstellen kann. Im Hinblick auf Archiv- und Dokumentationsprojekte sollte überlegt werden, wie eine Mischung aus verschiedenen Medien, z.B. verschiedenste schriftliche Dokumente sowie Video- und Audiomaterialien erstellt werden kann. Diese Überlegungen sollten von dem, was den besten Einblick in die Erfahrbarkeit von Praktiken gibt, und nicht von einem Zwang nach Vollständigkeit und der Angst vor Verlust, bestimmt sein. Die Entwicklung des modernen Tanzes ging einher mit der Sorge, dass das Ballett als Tanztechnik und damit auch eine gewisse »Objektivierung der Tanzkunst« verloren gehen könnte, deshalb, so Theodor W. Adorno, wurden in den dreißiger Jahren des vergangenen Jahrhunderts, in einem einzigen Jahrzehnt, mehr Tanzschriften entwickelt als in den Jahrhunderten zuvor (Adorno 2001). Doch Tanzkunst ist in erster Linie nicht objektivierbar, sondern sinnlich (sensual) erfahrbar. Deshalb kann sich die Darstellungsweise von Wissen über Tanz nicht in einer Übertragung in wissenschaftliche Sprache und/oder Tanznotation erschöpfen, eben weil dieses Wissen selbst sich so fundamental von Sprache unterscheidet und auch nicht objektivierbar ist. 


\section{Literatur}

Adorno, Theodor W. (2001): Zu einer Theorie der musikalischen Reproduktion, Frankfurt a.M.: Suhrkamp.

Bowie, Andrew (2009): Music, Philosophy and Modernity, Cambridge: Cambridge University Press.

https://charm.rhul.ac.uk/index.html [online] [14.01.2020]

Feyerabend, Paul (2010): Against Method, 4. Aufl. London/New York: Verso.

Horkheimer, Max/Theodor W. Adorno (1988): Die Dialektik der Aufklärung. Philosophische Fragmente, Frankfurt a.M.: Fischer.

Kant, Immanuel (2001): Kritik der Urteilskraft, Hamburg: Meiner.

Karnes, Kevin C. (2016): Music, Criticism, and the Challenge of History. Shaping Modern Musical Thought in Late Nineteenth-Century Vienna, Oxford/New York: Oxford University Press.

Melrose, Susan (2018): Nothing like ... falling ... , in: Performance Research, Jg. 23, Nr. 4-5, S. 152-161, DOI: 10.1080/13528165.2018.1511037

Merleau-Ponty, Maurice (1976): Die Phänomenologie der Wahrnehmung, Berlin: De Gruyter.

Reents, Friederike (2015): Stimmungsästhetik. Realisierungen in Literatur und Theorie vom 17. bis in 21. Jahrhundert, Göttingen: Wallstein.

Varela, Francesco J./Thompson, Evan/Rosch, Eleanor (1993): Cognitive Science and Human Experience, Massachusetts: MIT Press.

Zbikowski, Lawrence (2012): Music and Movement. A View from Cognitive Musicology, in: Stephanie Schroedter (Hg.), Bewegungen zwischen Sehen und Hören, Denkbewegungen über Bewegungskünste, Könighausen und Neumann: Würzburg, S. 151-162. 